\title{
Evaluation of Smagorinsky variants in large-eddy simulations of wall-resolved plane channel flows
}

\author{
Johan Meyers ${ }^{a)}$ \\ Department of Mechanical Engineering, Katholieke Universiteit Leuven, Celestijnenlaan 300A—bus 2421, \\ B3001 Leuven, Belgium \\ Pierre Sagaut ${ }^{\text {b) }}$ \\ Institut Jean Le Rond d'Alembert, Université Pierre et Marie Curie-Paris 6, Boîte 162, 4 place Jussieu, \\ 75252 Paris cedex 05, France
}

(Received 5 December 2006; accepted 11 July 2007; published online 19 September 2007)

\begin{abstract}
In recent years, variational multiscale (VMS) Smagorinsky models have emerged as new models for large-eddy simulations (LES). A common version is the small-small variant, which uses a small-scale extraction of the LES solution, obtained by high-pass filtering the resolved velocity field, to express a Smagorinsky term. The subsequent small-scale extraction of this term is used as a model. In the current work, three formulations of the small-small VMS model are investigated in large-eddy simulations of the plane channel flow. The basic small-small formulation (Model A) is modified to explicitly incorporate effects of the LES filter and the high-pass filter (Model B). A third modification (Model C) is further inertial-range consistent, allowing the use of constant model coefficients for filters widths which are situated in a finite Reynolds-number inertial subrange. We aim to evaluate the performance of these models in the presence of walls. Therefore, channel-flow simulations are performed for $\operatorname{Re}_{\tau}=110,300,400$, and 650. Further, the effect of changes in the shape of the high-pass filter used for the three models is investigated. A sharp cutoff filter and a Gaussian high-pass filter are considered. In addition, a range of high-pass-filter widths is included in the analysis. Evaluations of the skin-friction, mean-velocity profiles, Reynolds stresses, and spanwise velocity spectra are presented. We show that Model C is most insensitive to changes in Reynolds number and filter shape, closely followed by Model B. Model A is the most sensitive to the considered variations, and simulation quality depends in particular on variations in the filter shape. (C) 2007 American Institute of Physics. [DOI: 10.1063/1.2768944]
\end{abstract}

\section{INTRODUCTION}

Large-eddy simulation (LES) is a modern technique for the prediction of turbulent flows. In LES, large-scale turbulent eddies are explicitly represented in a transient flow simulation. However, in order to reduce computational cost to manageable levels, small-scale turbulence is not resolved, but modeled by a subgrid-scale closure.

LES and subgrid-scale modelling have been the subject of many studies in the last decades. ${ }^{1-4}$ The rationale for subgrid-scale modeling in LES is the observation that smallscale turbulence in high-Reynolds-number flows displays universal properties. Consequently, it is considered possible to represent the average effect of these small turbulent scales by means of a relatively simple subgrid-scale model. ${ }^{5}$ Various models have been designed with these principles in mind, ${ }^{1}$ and have been shown to work well in high-Reynoldsnumber equilibrium turbulence. ${ }^{6}$

A challenge for subgrid-scale models designed with universal equilibrium principles in mind, is the simulation of wall-bounded flows. The dominant turbulent structures in the "inner layer" of a boundary layer decrease with the Reynolds number, ${ }^{7}$ and are further strongly anisotropic. In wall-

\footnotetext{
${ }^{a)}$ Electronic mail: johan.meyers@mech.kuleuven.be

${ }^{b)}$ Electronic mail: sagaut@1mm.jussieu.fr
}

resolved LES, imposed by limited computer resources, these structures in the inner layer are resolved on relatively coarse grids. Hence, these conditions do not correspond to a typical LES filter cut in a turbulent universal equilibrium range.

A set of subgrid-scale models which have performed remarkably well in channel flows, are variational multiscale (VMS) Smagorinsky models. ${ }^{8}$ In the context of a variational multiscale analysis, Hughes et al. ${ }^{9}$ proposed a strict separation between the mean flow (and large scales of the solution), and a resolved small-scale subset of the LES field on which the model acts. In contrast to a standard Smagorinsky model, ${ }^{10}$ constant-coefficient VMS formulations of the Smagorinsky model were shown to yield accurate results both in homogeneous isotropic turbulence ${ }^{9,11}$ and in channel flows. ${ }^{8,12-14}$ However, the selection of model constants is still subject to empirical fits. ${ }^{13,14}$ The current paper presents a further evaluation of VMS Smagorinsky models, and aims at a reduction of the empiricism in the determination of their model constants. All discussion is based on the small-small formulation of the Smagorinsky model, ${ }^{9}$ which first expresses the Smagorinsky model using a small-scale extraction of the resolved field, and then takes a small-scale extraction of this result as a model for the subgrid-scale stresses.

In Ref. 15, a theoretical analysis was presented on VMS Smagorinsky coefficients required for the modeling of finiteReynolds-number locally homogeneous isotropic subgrid 
scales. Modifications were proposed to the original model formulations, which account for two effects. First of all, effects of the shape of the LES filter $\mathcal{G}$ and the high-pass filter $\mathcal{H}^{\prime}$ are explicitly expressed in the model formulation. Second, a modification to the underlying Smagorinsky model was introduced, which makes the model consistent in a finite-Reynolds number inertial subrange, yielding improved simulation results. ${ }^{16,17}$ In the current manuscript, we will investigate the effect of these modifications on plane channel flow simulations, and demonstrate that both filter-shape and finite-Reynolds-number effects play a role in channelflow LES with a VMS model. Therefore, next to the original small-small model (which we denote as Model A), a version including $\mathcal{G}$ and $\mathcal{H}^{\prime}$ filter-shape effects (Model B), and a fully inertial-range consistent version (Model C) are employed.

In order to provide challenging conditions for LES subgrid-scale closures in wall-resolved flows, the simulation resolutions are carefully selected. In particular, it has been documented that skin-friction and mean-profile predictions can be very accurate in coarse no-model simulations using specific combinations of streamwise and spanwise mesh resolutions. ${ }^{18}$ As we will briefly illustrate, these trends can appear when spanwise grids are too coarse to resolve the inner-layer large structures near the wall. Hence, for the evaluation of the Models A-C, coarse-grid simulation resolutions are selected which do resolve the large-scale structures at the wall. Associated no-model errors on the skin friction are, depending on the Reynolds number, $15 \%$ to $25 \%$.

First, we perform a calibration of the model coefficient at $\operatorname{Re}_{\tau}=300$, and show very accurate predictions of the skin friction, the mean velocity profile, Reynolds stresses, and the spanwise velocity spectra for the three models A-C. Subsequently, a comparison of Models A, B, and C is performed based on their robustness to changes in the Reynolds number, and the high-pass filter $\mathcal{H}^{\prime}$. Simulations at $\operatorname{Re}_{\tau}=110$, 300, 400, and 650 are included. Further, for $\mathcal{H}^{\prime}$, a sharp cutoff and a Gaussian filter are used at different filter widths. It is demonstrated that Model $\mathrm{C}$ is the least sensitive to changes in the Reynolds number, or in the filter shape $\mathcal{H}^{\prime}$, closely followed by Model B. Variations on results observed for Model A are considerably higher.

The paper is further organized as follows. In Sec. II the governing equations and computational setup are presented. Next, in Sec. III, three subgrid-scale-model variants are introduced. Channel-flow simulation results using these models are discussed in Sec. IV. Finally, conclusions are formulated in Sec. V.

\section{GOVERNING EQUATIONS AND SETUP}

We will briefly outline the computational setup, the governing equations and the numerical discretization employed. Particularities related to the subgrid-scale models, will be discussed in the next section.

The channel flow is contained in a box with size $L_{x}$ in the streamwise direction $x$, size $2 \delta$ in the normal direction $y$, and size $L_{z}$ in the spanwise direction $z$. No-slip walls are
TABLE I. Overview of different simulation Reynolds numbers. For sake of compactness, we will refer to these cases, respectively, as the $\operatorname{Re}_{\tau}=110,300$, 400 , and 650 cases.

\begin{tabular}{lcccc}
\hline \hline $\operatorname{Re}$ & 3220.4 & 10039.1 & 13924.9 & 24272.2 \\
$\operatorname{Re}_{\tau}$ & 109.43 & 297.90 & 395.76 & 642.54 \\
\hline \hline
\end{tabular}

located at $y=-\delta$ and $y=\delta$. The flow is artificially forced in streamwise direction with a volume forcing such that the massflow is constant. This allows the use of periodic boundary conditions in the streamwise direction and periodic boundary conditions are also used for the spanwise direction. During computations and for the presentation of results, we will select a nondimensional representation of the channel flow, such that $\delta=1$, and the mean streamwise velocity $\bar{U}=1$.

We include channel-flow simulations at various Reynolds numbers. An overview is presented in Table I. Here, the computational Reynolds number $\mathrm{Re}$ is defined as $\operatorname{Re}=2 \delta \bar{U} / \nu$, and $\operatorname{Re}_{\tau}=\delta u_{\tau} / \nu$ is the Reynolds number based on the friction velocity $\left(u_{\tau}=\sqrt{\tau_{w}}\right.$, with $\tau_{w}$ the mean shear at the wall). Direct numerical simulation (DNS) reference data are taken from Ref. 19. For sake of compactness, we will refer to the cases in Table I, respectively, as the $\operatorname{Re}_{\tau}=110$, 300,400 , and 650 cases.

Before introducing the governing equations, i.e., the filtered Navier-Stokes equations, it is common practice to define a low-pass filter operation $\mathcal{G}$ as

$$
\tilde{f}(\mathbf{x})=\mathcal{G} f=\int_{\mathcal{D}} K_{\mathcal{G}}\left(\mathbf{x}-\mathbf{x}^{\prime}\right) f\left(\mathbf{x}^{\prime}\right) \mathrm{d}^{3} \mathbf{x}^{\prime},
$$

where $K_{\mathcal{G}}(\mathbf{x})$ is the filter kernel defined for all $\mathbf{x}$. Further, $\mathcal{G}$ has the property that $\mathcal{G} c=c$, for every constant function $c$.

Based on the filter $\mathcal{G}$, the nondimensional filtered NavierStokes equations for incompressible flows are formally expressed as

$$
\begin{aligned}
& \frac{\partial \widetilde{u}_{j}}{\partial x_{j}}=0, \\
& \frac{\partial \widetilde{u}_{i}}{\partial t}+\frac{\partial \widetilde{u}_{i} \widetilde{u}_{j}}{\partial x_{j}}+\frac{\partial \widetilde{p}}{\partial x_{i}}-\frac{4}{\operatorname{Re}} \frac{\partial \widetilde{S}_{i j}}{\partial x_{j}}-\frac{\partial \tau_{i j}}{\partial x_{j}}=0,
\end{aligned}
$$

where $\widetilde{u}_{i}$ is the filtered velocity component in the $x_{i}$ direction, and $\widetilde{p}$ the filtered pressure $\left(\left[\widetilde{u}_{1}, \tilde{u}_{2}, \widetilde{u}_{3}\right]\right.$, and $\left[x_{1}, x_{2}, x_{3}\right]$ are used here as alternative for $[\tilde{u}, \widetilde{v}, \widetilde{w}]$, and $[x, y, z])$. Further, $\widetilde{S}_{i j}=\left[\partial \widetilde{u}_{i} / \partial x_{j}+\partial \widetilde{u}_{j} / \partial x_{i}\right] / 2$ corresponds to the filtered strain tensor, and Re is the computational Reynolds number defined before. The filtering of the Navier-Stokes equations gives rise the subgrid-scale stress tensor $\tau_{i j}$ that is unclosed, and given by

$$
\tau_{i j}=\tilde{u}_{i} \tilde{u}_{j}-\widetilde{u_{i} u_{j}} .
$$

In large-eddy simulations, these subgrid-scale stresses are replaced by a model $m_{i j}$, which aims to approximate their dynamic effect, and is based on the resolved velocity field $\widetilde{u}_{i}$ 
only. The different models $m_{i j}$ used in the current study are introduced in the next section.

The governing equations (2) and (3) are discretized in streamwise $x$ and spanwise $z$ directions based on a pseudospectral Fourier method, and de-aliasing is performed using the two-third de-aliasing rule. Fourier transforms are implemented using the FFTW library. ${ }^{20}$ The wall-normal direction is discretized using a fourth-order energy-conserving staggered finite-volume discretization; ${ }^{21}$ i.e., the locations of wall-normal velocities $\widetilde{v}$ are shifted in the wall-normal direction by half a cell compared to $\widetilde{p}, \tilde{u}$, and $\widetilde{w}$. Continuity is retained by solving a Poisson equation for the pressure, using a direct solver, which is based on a Cholesky factorization. The time integration is performed by a classical four-stage fourth-order accurate Runge-Kutta time integration. ${ }^{22}$ Time steps are restricted by setting both the convective and viscous Courant-Friedrichs-Lewy number to 0.1 , which we selected well below the stability requirements in order to exclude time-integration errors.

The grid is uniform in the $x$ and $z$ directions. In the wall-normal direction, a mapping is used which maps a computational coordinate $\xi \in[-1,1]$ to $y \in[-\delta, \delta]$ using $y=a \tanh (b \xi)$. The parameters $a$ and $b$ are fixed by the number of grid points and the desired width of the first cell near the wall. Further details on the selected simulation grids are given in Sec. IV.

All simulations are started from a laminar velocity profile, superimposed with divergence-free random perturbations. After a sufficiently long transient, statistical averaging is started until the skin friction, the mean profiles, and velocity spectra are converged.

It is common practice to present channel-flow simulation results in wall units, i.e. normalized with the friction velocity. To this end, either the DNS reference friction velocity, or the LES prediction of the friction velocity may be employed. Since both approaches will be useful for the presentation of results, we mark properties normalized with the DNS friction velocity $u_{\tau, \text { DNS }}$ with a “*” superscript, and properties normalized with the LES friction velocity $u_{\tau, \text { LES }}$ with a "+" superscript.

\section{SMAGORINSKY VARIANTS}

We now introduce the subgrid-scale models which are employed in the current study, and discuss their main features.

First, the small-scale extraction of a property $f(\mathbf{x})$ is defined as

$$
f^{\prime}(\mathbf{x})=\mathcal{H}^{\prime} f=\int_{\mathcal{D}} K_{\mathcal{H}^{\prime}}\left(\mathbf{x}-\mathbf{x}^{\prime}\right) f\left(\mathbf{x}^{\prime}\right) \mathrm{d}^{3} \mathbf{x}^{\prime} .
$$

$\mathcal{H}^{\prime}$ is a high-pass convolution filter, and is either a projection operator, as originally introduced by Hughes et al., ${ }^{9}$ or, e.g., a smooth filter. ${ }^{23-25}$ In contrast to $\mathcal{G}$, the application of $\mathcal{H}^{\prime}$ to a constant function yields $\mathcal{H}^{\prime} c=0$.

The small-small VMS Smagorinsky model, is now formulated as (also referred to as Model A)

$$
m_{i j}^{A}=-2\left[\frac{\left(C_{S} \Delta\right)^{2}}{\left(1-\beta^{4 / 3}\right)^{3 / 2}}\left|\widetilde{S}^{\prime}\right| \tilde{S}_{i j}^{\prime}\right]^{\prime},
$$

with $C_{s}$ the model coefficient, and $\beta=\Delta / \Delta^{\prime}$ the ratio of the $\mathcal{G}$-filter width to the $\mathcal{H}^{\prime}$-filter width. The factor $\left(1-\beta^{4 / 3}\right)^{-3 / 4}$ accounts for the difference between the standard Smagorinsky formulation, which employs all resolved LES scales, and the small-small model, which uses a small-scale part of the resolved field in its formulation. Using Lilly's analysis, ${ }^{26}$ Hughes et al. ${ }^{11}$ introduced this scaling, and further identified $C_{s}=C_{s, \infty}$, where

$$
C_{s, \infty}=\frac{1}{\pi}\left(\frac{2}{3 \alpha}\right)^{3 / 4}
$$

is the Lilly Smagorinsky constant, and $\alpha$ the Kolmogorov constant $(\alpha \approx 1.5)$.

In Eq. (6), $\left|\tilde{S}^{\prime}\right|$ is the magnitude of the small-scale extracted strain tensor, which we take

$$
\left|\widetilde{S}^{\prime}\right|=\left(\left\langle 2 \widetilde{S}_{i j}^{\prime}{\widetilde{S_{i j}}}_{i j}^{\prime}\right\rangle_{x, z}\right)^{1 / 2}
$$

with $\langle\cdot\rangle_{x, z}$ averaging over wall-parallel $x-z$ planes, such that implementation of the models in the channel code does not require the use of Fourier transforms to calculate the product $\tilde{S}_{i j}^{\prime} \widetilde{S}_{i j}^{\prime}$. Moreover, the filter width $\Delta$ is a length scale which we will assume to be related to the grid cutoff, and it is common practice to take ${ }^{27}$

$$
\Delta=\left(h_{x} h_{y} h_{z}\right)^{1 / 3},
$$

with $h_{x}, h_{y}$, and $h_{z}$ the local grid spacing in $x, y$, and $z$ directions.

In Ref. 15, an extensive theoretical analysis was presented on the VMS Smagorinsky model coefficients, and a set of parameters was identified, which dictate the required model coefficients. Here, we briefly discuss these parameters (i-iv) in the context of wall-bounded flows, and some of them will be incorporated into two evolutions of Model A, denoted Models B and C.

(i) The shape of the LES filter $\mathcal{G}$ has a profound effect on the model coefficient. Often, $\mathcal{G}$ is assumed to be a "grid cutoff" filter, and we will follow this in the present study. A more involved analysis, which would include the implicit $\mathcal{G}$-filter shape related to the smallsmall Smagorinky model, similar to proposals for the implicit Smagorinsky filter, ${ }^{7,28,29}$ is not in the scope of the current paper.

(ii) The shape of the high-pass filter $\mathcal{H}^{\prime}$ is explicitly defined in the formulation of the small-small formulation. The combined effect of $\mathcal{G}$ and $\mathcal{H}^{\prime}$ can be incorporated in the formulation of the small-small model. ${ }^{15}$ In the context of wall bounded flows, this approach has to be extended for inhomogeneous grids. This will be elaborated in Sec. III A, leading to Model B.

(iii) At finite Reynolds numbers, a filter width in the inertial-subrange does not lead to a constant model coefficient $C_{s}{ }^{15}$ The coefficient depends on the ratio $\Delta / \eta$, with $\eta$ the Kolmogorov scale. Lilly's model constant is only recovered for $\Delta / \eta=\infty$. In a channel- 
flow boundary layer, it is well documented ${ }^{30}$ that $\eta^{+} \sim\left(y^{+}\right)^{1 / 4}$, while the integral length scale $\ell^{+} \sim y^{+}$. Since the filter width $\Delta$ in LES will rather scale with the integral length scale $\ell$ than with the Kolmogorov scale $\eta$, it is clear that $\Delta / \eta$ depends on $y^{+}$[e.g., if $\Delta \sim \ell$, then $\left.\Delta / \eta \sim\left(y^{+}\right)^{3 / 4}\right]$. Consequently, the model coefficient in Model A also depends on $y^{+}$. In Ref. 15 a simple modification of the small-small model has been proposed, which facilitates the use of a constant model coefficient for finite $\Delta / \eta$ ratios. We will briefly describe this inertial-range consistent formulation of the small-small model in Sec. III B (Model C).

(iv) Finally, at low resolution of the integral-length scale, the model coefficients of the standard and variational multi-scale Smagorinsky models depend on the ratio $R=\ell / \Delta .{ }^{15}$ For $R \gg 1$, the model coefficients are independent of $R$, but the rate of convergence to this asymptotic value depends on the model, and the largescale flow features. In channel flows, the coarse resolutions used to resolve the dominant turbulent structures in the inner layer close to the wall, and the strong anisotropy of the turbulence in this region, may well yield a " $R$-dependence" of the model coefficient $C_{s}$.

These four items (i-iv) all have an effect on the performance of Model A, and may explain the need to tune the model coefficient $C_{s}$. Modifications to Model A are proposed in Sec. III A (Model B) and Sec. III B (Model C). Model B accounts for (i-ii), and Model $\mathrm{C}$ accounts for (i-iii).

The effect of (iv) is not straightforwardly incorporated in either of the formulations. Some uncertainties may also exist on the shape of $\mathcal{G}$ (i). Therefore, we will calibrate the coefficients $C_{s}$ in the three models. The sensitivity of the models to variations in $\mathcal{H}^{\prime}$ and the Reynolds number is then investigated, while keeping $\mathcal{G}$ and $R$ effects constant. This is further expounded in Sec. IV.

\section{A. Accounting for the shape of $\mathcal{G}$ and $\mathcal{H}^{\prime}$}

For homogeneous filters, it was demonstrated that the shape of $\mathcal{G}$ and $\mathcal{H}^{\prime}$ can be accounted for by adapting the model coefficient with a correction factor. ${ }^{15}$ Here, we will extend this analysis to inhomogeneous filters, which will, for the channel flow, yield $y$-dependent factors.

To account for the shape of $\mathcal{G}$ and $\mathcal{H}^{\prime}$, Model A can be generalized by including a correction factor $\gamma_{\beta}$ in the formulation (i.e., Model B)

$$
m_{i j}^{B}=-2\left[\frac{\left(C_{s} \Delta / \gamma_{\beta}\right)^{2}}{\left(1-\beta^{4 / 3}\right)^{3 / 2}}\left|\tilde{S}^{\prime}\right| \tilde{S}_{i j}^{\prime}\right]^{\prime}
$$

with $C_{s}$ a model constant. Under asymptotic conditions, i.e., $\operatorname{Re}=\infty$ and $R \gg 1$, the coefficient $C_{s}=C_{s, \infty}$, and $\gamma_{\beta}$ is a factor that depends only on the shapes of $\mathcal{G}$ and $\mathcal{H}^{\prime}$, as we will demonstrate next for filters with one inhomogeneous direction (i.e., the $y$ direction).

Under asymptotic conditions, with $C_{s}=C_{s, \infty}$, the total turbulent dissipation is expressed as

$$
\begin{aligned}
\varepsilon=\left\langle m_{i j}^{B} S_{i j}\right\rangle & =\left\langle 2\left[\frac{\left(C_{s, \infty} \Delta / \gamma_{\beta}\right)^{2}}{\left(1-\beta^{4 / 3}\right)^{3 / 2}}\left|\widetilde{S}^{\prime}\right| \widetilde{S}_{i j}^{\prime}\right]^{\prime} \widetilde{S}_{i j}\right\rangle \\
& =\left\langle 2 \frac{\left(C_{s, \infty} \Delta / \gamma_{\beta}\right)^{2}}{\left(1-\beta^{4 / 3}\right)^{3 / 2}}\left|\widetilde{S}^{\prime}\right| \widetilde{S}_{i j}^{\prime} \widetilde{S}_{i j}^{\prime}\right\rangle \\
& =\frac{\left(C_{s, \infty} \Delta / \gamma_{\beta}\right)^{2}}{\left(1-\beta^{4 / 3}\right)^{3 / 2}}\left\langle 2 \widetilde{S}_{i j}^{\prime} \widetilde{S}_{i j}^{\prime}\right)^{3 / 2} .
\end{aligned}
$$

Here, we used $\left\langle f^{\prime} g\right\rangle=\left\langle f g^{\prime}\right\rangle$, which is valid for self-adjoint filters $\mathcal{H}^{\prime}$, using $\langle\cdot\rangle$ as the related inner product. ${ }^{15,25}$ The filters which are used in the present study comply with this property.

If the LES is well resolved, i.e., $R \gg 1$, such that the filter cuts in the inertial subrange, one can express the ensemble $\left\langle\widetilde{S}_{i j}^{\prime} \widetilde{S}_{i j}^{\prime}\right\rangle$ based on the velocity-spectrum tensor; i.e., ${ }^{7}$

$$
\left\langle\widetilde{S}_{i j}^{\prime} \widetilde{S}_{i j}^{\prime}\right\rangle=\frac{1}{2} \iiint k^{2} \Phi_{i i}(\mathbf{k})\left[G(\mathbf{k}) H^{\prime}(\mathbf{k})\right]^{2} \mathrm{~d}^{3} \mathbf{k} .
$$

$G(\mathbf{k})$ and $H^{\prime}(\mathbf{k})$ are the transfer functions associated with $\mathcal{G}$ and $\mathcal{H}^{\prime}$, and $k$ is the magnitude of the wavevector $\mathbf{k}=\left[k_{1}, k_{2}, k_{3}\right]$. Further,

$$
\Phi_{i j}(\mathbf{k})=\frac{1}{4 \pi k^{2}}\left(\delta_{i j}-\frac{k_{i} k_{j}}{k^{2}}\right) E(k) .
$$

Here, $\delta_{i j}$ represents the Kronecker delta, and $E(k)$ $=\alpha \varepsilon^{2 / 3} k^{-5 / 3}$ is an idealized inertial-range energy spectrum, which can be employed since $R \gg 1$ and $\operatorname{Re}=\infty$ are assumed.

Combination of Eqs. (11)-(13), leads to

$\gamma_{\beta}$

$$
=\left(\frac{1}{3 \pi} \frac{\int_{-\pi / h_{x}}^{\pi / h_{x}} \int_{-\pi / h_{y}}^{\pi / h_{y}} \int_{-\pi / h_{z}}^{\pi / h_{2}} k^{-5 / 3}\left[H^{\prime}(\mathbf{k}) G(\mathbf{k})\right]^{2} \mathrm{~d} k_{x} \mathrm{~d} k_{y} \mathrm{~d} k_{z}}{(\pi / \Delta)^{4 / 3}\left(1-\beta^{4 / 3}\right)}\right)^{3 / 4},
$$

where we further used the grid cutoff wavenumbers in the three directions as integration bounds for the integrals.

Based on Eq. (14), it is readily verified that $\gamma_{\beta}=1$, provided following conditions are satisfied: $\mathcal{G}$ is a spherical sharp cutoff filter with cutoff $k_{c}=\pi / \Delta, h_{x}=h_{y}=h_{z}$, and $\mathcal{H}^{\prime}$ is a spherical (high-pass) cutoff filter with cutoff $k_{c}^{\prime}=\beta \pi / \Delta$. For these conditions $\left(\gamma_{\beta}=1\right)$, Models B and A are equivalent.

For the current study, we take $\mathcal{G}$ the grid cutoff filter, with cutoff wavenumber $\mathbf{k}_{c}=\left[\pi / h_{x}, \pi / h_{y}, \pi / h_{z}\right]$. Since $\mathbf{k}_{c}$ is explicitly used in the integration bounds in Eq. (14), this corresponds to selecting $G(\mathbf{k})=1$ in Eq. (14).

When $\beta \rightarrow 0$, Model B converges to a standard Smagorinsky model. Using $\beta=0$ and taking $\mathcal{G}$ the grid cutoff filter, we verified that $\gamma_{0}$ is equivalent with the correction factor introduced by Scotti et $a l .{ }^{31}$ for the Smagorinsky model on anisotropic grids.

Two different shapes for the high-pass filters $\mathcal{H}^{\prime}$ are investigated in the current work. For ease of implementation, these filters act only in wall-parallel planes (VMS Smagorinsky simulations using wall-normal high-pass filters may be found in Ref. 14). The first filter is a square sharp cutoff filter defined as 


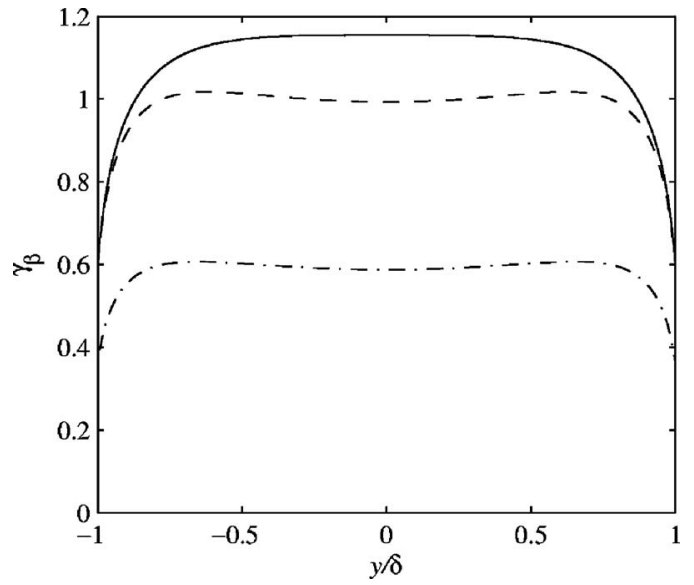

FIG. 1. The factor $\gamma_{\beta}$ for different selections of $\mathcal{H}^{\prime}$ on a $48^{3}$ grid. The selected mesh corresponds to $h_{x}=0.16 \delta, h_{z}=0.065 \delta, h_{y_{w}}=\delta / 500$, and $h_{y}$ $=0.049 \delta$ (cf. also Table II). $\mathcal{G}$ corresponds to the grid cutoff filter, and $\mathcal{H}^{\prime}$ corresponds to (-): $\beta=0,(--)$ : A wall-parallel square cutoff filter with $\beta$ $=0.5$, and (-.) a wall-parallel Gaussian filter with $\beta=0.5$.

$$
\begin{aligned}
H^{\prime}(\mathbf{k})= & 1-\left[\mathbf{H}\left(k_{x},-\frac{\pi \beta}{h_{x}}\right)-\mathbf{H}\left(k_{x}, \frac{\pi \beta}{h_{x}}\right)\right] \\
& \times\left[\mathbf{H}\left(k_{z},-\frac{\pi \beta}{h_{z}}\right)-\mathbf{H}\left(k_{z}, \frac{\pi \beta}{h_{z}}\right)\right],
\end{aligned}
$$

with $\mathbf{H}(x, a)$ the Heaviside step function $(\mathbf{H}(x, a)=0, x<a$, and $\mathbf{H}(x, a)=1, x \geqslant a)$. The second filter is a Gaussian filter defined as

$$
H^{\prime}(\mathbf{k})=1-\exp \left(-\frac{\left(k_{x} h_{x}\right)^{2}+\left(k_{z} h_{z}\right)^{2}}{24 \beta^{2}}\right) .
$$

In Fig. 1 , an example is presented of $\gamma_{\beta}$ for these two filters and $\beta=0.5$ as function of the wall-normal direction $y$ on a $48^{3}$ mesh. Further, $\gamma_{0}$, selecting $\mathcal{H}^{\prime}$ the identity operator (and $\beta=0$ ), is also presented. To this end, Eq. (14) is evaluated using standard numerical integration techniques. The singularity that occurs at $k_{x}=k_{y}=k_{z}=0$ is easily circumvented by recasting the integrals in Eq. (14) for a small zone around $k=0$ into a spherical coordinate frame. Figure 1 clearly illustrates the dependence of $\gamma_{\beta}$ on $\mathcal{H}^{\prime}$, the grid cutoff filter $\mathcal{G}$, and $\beta$.

\section{B. An inertial-range consistent formulation}

In Ref. 15 an analysis was presented on the model coefficients associated with variational multiscale Smagorinsky models in finite-Reynolds-number isotropic turbulence. For LES filters in the inertial subrange, it was shown that VMS model coefficients depend on the ratio of the filter with $\Delta$ to the Kolmogorov scale $\eta$. In order to circumvent an explicit $\Delta / \eta$ dependence of model coefficients, modified models were proposed, of which the model coefficients do not depend on $\Delta / \eta$ in the inertial subrange. For the small-small model, a possible inertial-range consistent formulation corresponds to ${ }^{15}$ (Model C)

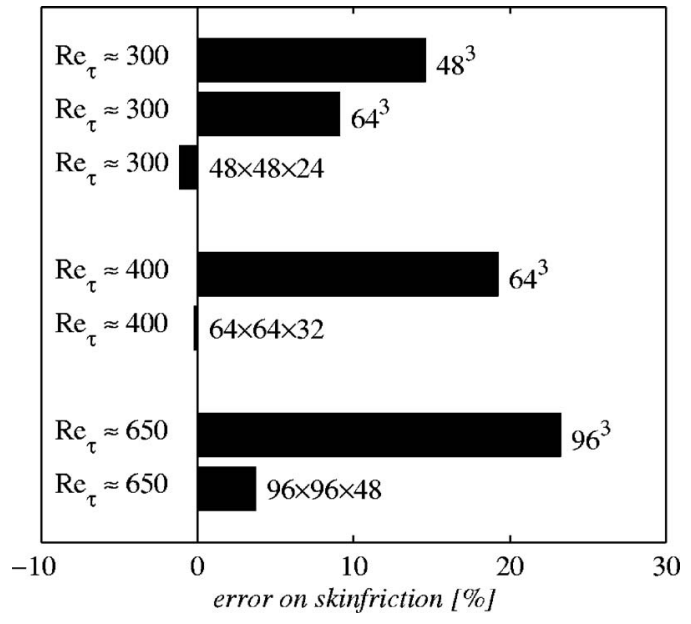

FIG. 2. Relative errors on the skin friction for various no-model simulations.

$$
\begin{aligned}
m_{i j}^{C}= & -2\left[\frac{\left(\gamma_{0} / \gamma_{\beta}\right)^{4 / 3}}{1-\beta^{4 / 3}}\right. \\
& \left.\times\left(\sqrt{\left(\frac{C_{s} \Delta}{\gamma_{0}}\right)^{4} \frac{\left(\gamma_{0} / \gamma_{\beta}\right)^{4 / 3}\left|\tilde{S}^{\prime}\right|^{2}}{1-\beta^{4 / 3}}+\nu^{2}}-\nu\right) \widetilde{S}_{i j}^{\prime}\right]^{\prime},
\end{aligned}
$$

with $\nu=2 / \operatorname{Re}$ the molecular viscosity. Under asymptotic conditions, i.e., $R \gg 1$, one finds $C_{s}=C_{s, \infty}$. Differently from Models $\mathrm{A}$ and $\mathrm{B}, \mathrm{Re}=\infty$ is not required for this.

\section{CHANNEL FLOW SIMULATION RESULTS}

An overview of channel-flow results for a range of Reynolds numbers and different high-pass filters $\mathcal{H}^{\prime}$ is presented for the models A-C. First of all, based on an evaluation of no-model simulations, a selection of relevant resolutions is made in Sec. IV A. Next, a set of calibration runs is performed for $\operatorname{Re}_{\tau}=300$, as will be further discussed in Sec. IV B. Subsequently, the effect of the shape of $\mathcal{H}^{\prime}$, and the ratio $\beta$, are studied in Sec. IV C. Finally, the Reynolds number is varied, i.e., $\operatorname{Re}_{\tau}=110-650$ in Sec. IV D.

\section{A. Selection of simulation resolutions}

In order to come to a selection for the simulation resolution, we performed a set of no-model simulations. Based on these results minimum simulation resolutions were identified, which are "as challenging as possible" for LES, without under-resolving the inner layer. We will highlight some of these simulations, briefly discuss convergence trends, and isolate some solution types which appear relatively good on first sight, but are to be avoided in wall-resolved LES.

In Fig. 2, the relative error on the skin friction $\tau_{w}$ is presented for a selection of resolutions, where

$$
\tau_{w}=\left.\frac{2}{\operatorname{Re}} \frac{\partial U}{\partial y}\right|_{y=0},
$$

and $U=\langle u\rangle$ is the ensemble averaged streamwise velocity profile. The relative error is straightforwardly defined as $\delta_{\tau}=\left(\tau_{w, \mathrm{LES}}-\tau_{w, \mathrm{DNS}}\right) / \tau_{w, \text { DNS }}$. First of all, for the $\operatorname{Re}_{\tau}=300$ 
TABLE II. Overview of the simulation grid sizes expressed in wall units for all Reynolds-number cases.

\begin{tabular}{lcccccc}
\hline \hline $\operatorname{Re}_{\tau}$ & $h_{x}^{+}$ & $h_{z}^{+}$ & $h_{y_{w}^{+}}$ & $h_{y_{c}}^{+}$ & $L_{x} \times L_{y} \times L_{z}$ & $N^{3}$ \\
\hline 110 & 53.7 & 21.5 & 0.71 & 8.1 & $5 \pi \delta \times 2 \delta \times 2 \pi \delta$ & $32^{3}$ \\
300 & 48.7 & 19.5 & 1.25 & 14.5 & $2.5 \pi \delta \times 2 \delta \times \pi \delta$ & $48^{3}$ \\
400 & 48.6 & 19.4 & 1.64 & 13.4 & $2.5 \pi \delta \times 2 \delta \times \pi \delta$ & $64^{3}$ \\
650 & 52.6 & 21.0 & 1.97 & 14.0 & $2.5 \pi \delta \times 2 \delta \times \pi \delta$ & $96^{3}$ \\
\hline \hline
\end{tabular}

case, the errors related to the $48^{3}$ and $64^{3}$ no-model simulations behave as expected, and the $48^{3}$ resolution is a good candidate for further model testing. The error-level for the no-model $48 \times 48 \times 24$ simulation is out of line. As we will further demonstrate, this resolution does not properly resolve the inner layer. In the selected case this leads to an apparent high-quality channel-flow simulation with a very low error on the skin friction. ${ }^{18}$

In Fig. 2, some results are also presented for $\operatorname{Re}_{\tau}=400$, and $\operatorname{Re}_{\tau}=650$. The respective resolutions $64^{3}$ and $96^{3}$ correspond to the $48^{3}$ resolution in the $\operatorname{Re}_{\tau}=300$ case, selected such that the resolution of the inner layer remains constant. This is further illustrated in Table II, where the grid sizes for the different $\operatorname{Re}_{\tau}$ cases are displayed in wall units. Further, it is clear in Fig. 2 that the $64 \times 64 \times 32$, and $96 \times 96 \times 48$ resolutions for the $\operatorname{Re}_{\tau}=400$, and $\operatorname{Re}_{\tau}=650$ case show the same low skin-friction error as observed previously. This is related to the same under-resolution of the inner layer, i.e., with $\Delta z^{+} \approx 40$, which is too high to correctly resolve the typical near-wall streaks. ${ }^{1}$

The results of the no-model $\operatorname{Re}_{\tau}=300$ runs are briefly assessed. First of all, in Fig. 3 the mean streamwise velocity profiles are compared to the DNS results. As expected the $64^{3}$ profile is closer to the DNS than the $48^{3}$, but again, it is the $48 \times 48 \times 24$ simulation that provides the best fit to the DNS results. The slope of this $48 \times 48 \times 24$ profile is not correct, but one might be tempted to try and "correct" the relative small deviation from the DNS profile by means of a subgrid-scale model.

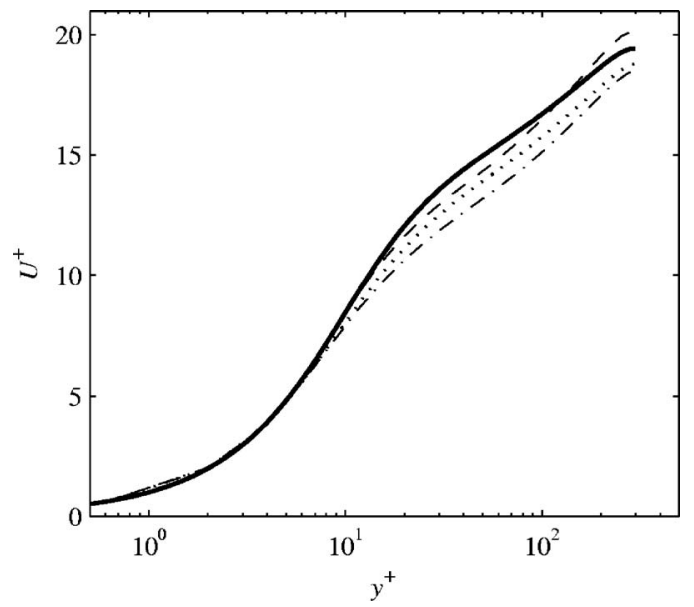

FIG. 3. Mean streamwise velocity profiles for $\operatorname{Re}_{\tau}=300$, and the reference DNS (-), the $48^{3}(-), 64^{3}(\cdots)$, and $48 \times 48 \times 24(--)$ no-model simulations.
Therefore, it is instructive to look, in the inner layer close to the wall, at the one-dimensional spanwise spectra $E_{y z}$ associated with the normal velocity $v$. In Fig. 4 these spectra are displayed for the different $\operatorname{Re}_{\tau}=300$ simulations. Now, the under resolution of the $48 \times 48 \times 24$ in the spanwise direction $z$ is apparent. For this simulation, the energycontaining range of the spectrum is cut by the mesh cutoff filter. Clearly, all three no-model simulations overpredict the level of the energy spectrum. For the $48^{3}$ and $64^{3}$ runs, this yields a high level of turbulent energy close to the wall, which leads to an overprediction of the skin friction. In the $48 \times 48 \times 24$ case, the high level of the spectrum does not reflect in an overall high energy of the turbulent structures close to the wall, since a significant part of the spectrum is not represented on the grid. For this case, the skin-friction prediction is considerably lower. In the current paper, we will next to skin friction and mean profiles, also evaluate Reynolds-stress predictions and spanwise normal-velocity spectra to ascertain that this type of mechanisms do not occur in our selected large-eddy simulations.

\section{B. $\mathbf{R e}_{\tau}=\mathbf{3 0 0}$ reference simulations}

We now turn to LES using Models A, B, and C respectively defined in Eqs. (6), (10), and (17).

In order to circumvent uncertainties on the shape of the LES filter $\mathcal{G}$, and on $R=\ell / \Delta$ [cf. Sec. III, items (i) and (iv)], $C_{s}$ is calibrated for the $\operatorname{Re}_{\tau}=300$ case, such that errors on the skin friction are low. To this end, we select $\mathcal{H}^{\prime}$ a sharp cutoff filter [Eq. (15)], and $\beta=0.5$. Subsequently, we try to keep

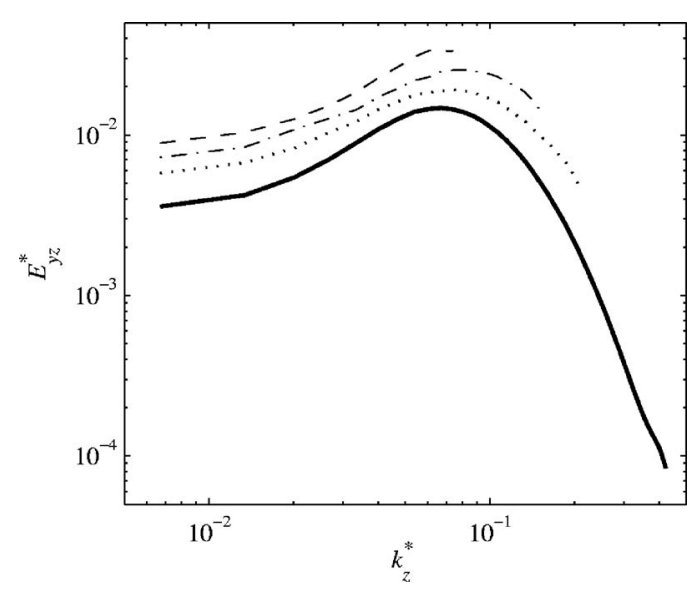

FIG. 4. Spanwise normal-velocity spectra $E_{y z}$ at $y^{*} \approx 15$ for $\operatorname{Re}_{\tau}=300$, and the reference DNS (-), the $48^{3}(-), 64^{3}(\cdots)$, and $48 \times 48 \times 24(--)$ nomodel simulations. 


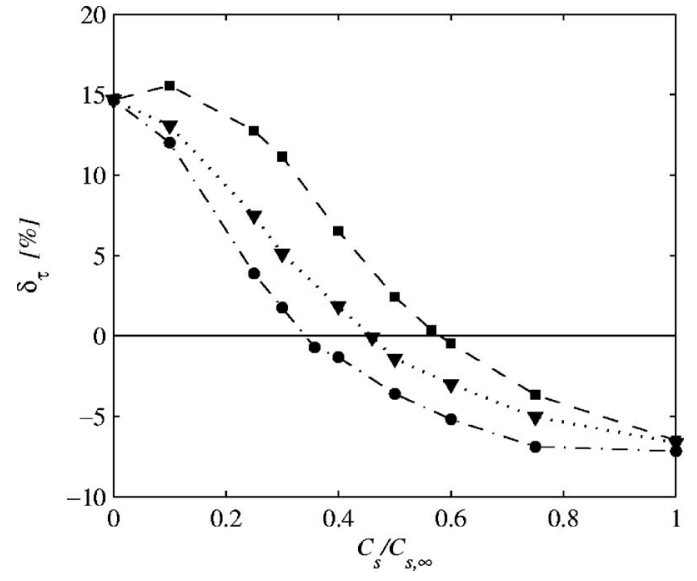

FIG. 5. Error on skin friction $\left(\operatorname{Re}_{\tau}=300\right)$ as function of the model coefficient $C_{s} .(\cdots \nabla)$ : Model A; $(-\bullet \bullet)$ : Model B; and $(--\mathbf{\square})$ : Model C.

effects of $\mathcal{G}$, and $R$ constant in the next sections, while the sensitivity of the models to variations in $\operatorname{Re}_{\tau}, \beta$, and $\mathcal{H}^{\prime}$ is evaluated

In Fig. 5, the dependence of the error on the skin friction $\delta_{\tau}$ is presented for the three models $\mathrm{A}, \mathrm{B}$, and $\mathrm{C}$, all employing a sharp cutoff high-pass filter $\mathcal{H}^{\prime}$ [Eq. (15)] with $\beta=0.5$. Clearly, $C_{s}=C_{s, \infty}$ is not an accurate option for any of the considered models. Instead, calibrated values for $C_{s}$, which give a low error on the skin friction (i.e., $<0.5 \%$ ) correspond to $C_{s}^{A} \approx 0.46 C_{s, \infty}, C_{s}^{B} \approx 0.36 C_{s, \infty}$, and $C_{s}^{C} \approx 0.66 C_{s, \infty}$ for Models $\mathrm{A}, \mathrm{B}$, and $\mathrm{C}$, respectively. These constants will be used for further evaluation of the different models.

First of all, the quality of the calibrated results is further established. Predictions of the mean streamwise velocity profiles $U(y)$ are displayed in Fig. 6. It is appreciated that all three models provide good mean-velocity profiles, and the improvement compared to the no-model case is considerable.

In Figs. 7(a)-7(d), the $u^{*} u^{*}, v^{*} v^{*}, w^{*} w^{*}$, and $-u^{*} v^{*}$ Reynolds-stress results are shown. In order to facilitate the interpretation of no-model stresses, we chose here to present "*-normalized" results, such that incorrect no-model predictions of the stresses are not concealed by an incorrect nomodel friction velocity. For the three fitted models, virtually no differences exist between "+-normalization" and "*normalization," since $u_{\tau}$ is predicted with an error lower than $0.25 \%\left(\delta_{\tau}<0.5 \%\right)$

In the DNS reference database, ${ }^{19}$ only unfiltered data are available, and these are used in Fig. 7. Hence, a comparison with LES data is strictly speaking not correct, ${ }^{32}$ and mainly qualitative comparisons are made. Based on the available DNS spectra ${ }^{19}$ we estimate that the difference between filtered and unfiltered levels of $u u$, and $w w$, is lower then $3 \%$. For $v v$ the relative difference between filtered and unfiltered values is higher; i.e., $5 \%$ at the channel center and up to $20 \%$ close to the wall (where $v v$ approaches zero $\sim y^{4}$ ).

In Fig. 7(a), the $u u$ stresses are shown. The improvement of the results compared to the no-model simulations is apparent, and can be situated mainly in the range $50<y^{*}$ $<250$. All three models $(\mathrm{A}-\mathrm{C})$ give nearly identical results. In Fig. 7(b), roughly the same trends are observed, but now

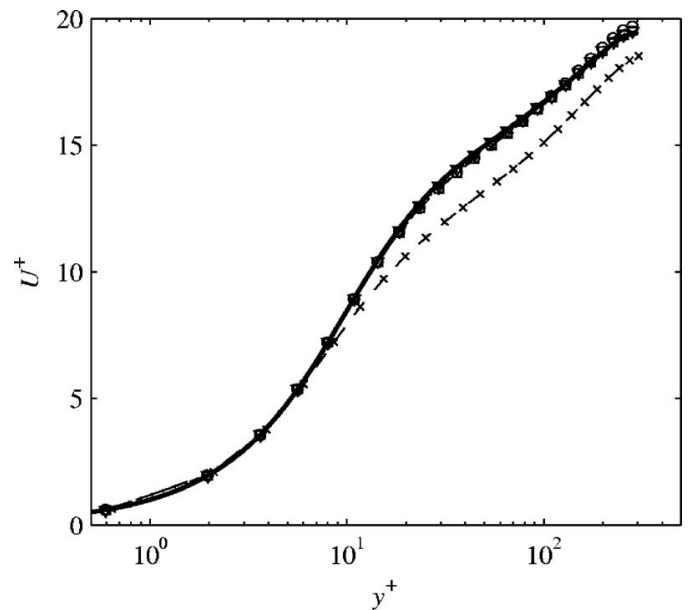

FIG. 6. Mean velocity profile for the fitted models and the no-model simulation at $\operatorname{Re}_{\tau}=300$. (-): DNS reference; $(--\times)$ : no model; $(\cdots \nabla)$ : Model A; (-.o): Model B; and (-- $\square)$ : Model C.

the improvement over the no-model simulation is most dominantly visible in the range $0<y^{*}<100$. Likewise, the three models (A-C) improve the prediction of $w w$ in Fig. 7(c) compared to the no-model case. Finally, the prediction of $u v$ in Fig. 7(d) by the three models is excellent.

The normal-velocity spanwise spectrums at $y^{*} \approx 15$ are shown in Fig. 8. Improvement over the no-model case is clear. All three models provide nearly identical results, which are very accurate for $k_{z}^{*}>8$. However, at $k_{z}^{*} \approx 8$, there is a discontinuity in the spectra, which corresponds with the location of the high-pass cutoff wavenumber $k_{c}^{\prime}=\beta \pi / h_{z}$. Hence, the sharp separation between the resolved small-scale part where the models act, and the large-scale part where they do not dissipate, is visible in the solution. In homogeneous isotropic turbulence, similar observations were reported. ${ }^{33}$ Furthermore, for $k_{z}^{*}<8$, the shape of the spectrum is reasonably well predicted, but the prediction is slightly too high.

\section{Variation of the high-pass filter $\mathcal{H}^{\prime}$}

The effect of changes to the high-pass filter $\mathcal{H}^{\prime}$ are now investigated. As discussed before [cf. issue (ii) in Sec. III], the high-pass filter may have a considerable effect on the selection of the model coefficient for Model A. Hence, keeping the coefficient to its calibrated value, while changing the $\mathcal{H}^{\prime}$ filter, may have a significant effect on the models performance. Models B and $\mathrm{C}$ include an explicit $\mathcal{H}^{\prime}$-filter dependence, which should rend their respective performance insensitive to $\mathcal{H}^{\prime}$ variations. We will investigate this by changing the shape of $\mathcal{H}^{\prime}$, and by changing the ratio $\beta=\Delta / \Delta^{\prime}$. $\operatorname{Re}_{\tau}=300$ is used throughout.

First of all, the shape of $\mathcal{H}^{\prime}$ is altered, and next to a sharp cutoff filter [Eq. (15)], a Gauss filter is considered [Eq. (16)], both with $\beta=0.5$. In Fig. 9, the relative errors on the skin friction $\delta_{\tau}$ are shown for Models $\mathrm{A}, \mathrm{B}$, and $\mathrm{C}$, using these two high-pass filters and $\beta=0.5$. Model $\mathrm{C}$ is the least sensitive to variations in filter shape (variation of $0.16 \%$ ), closely followed by Model B (variation of $0.74 \%$ ). In con- 

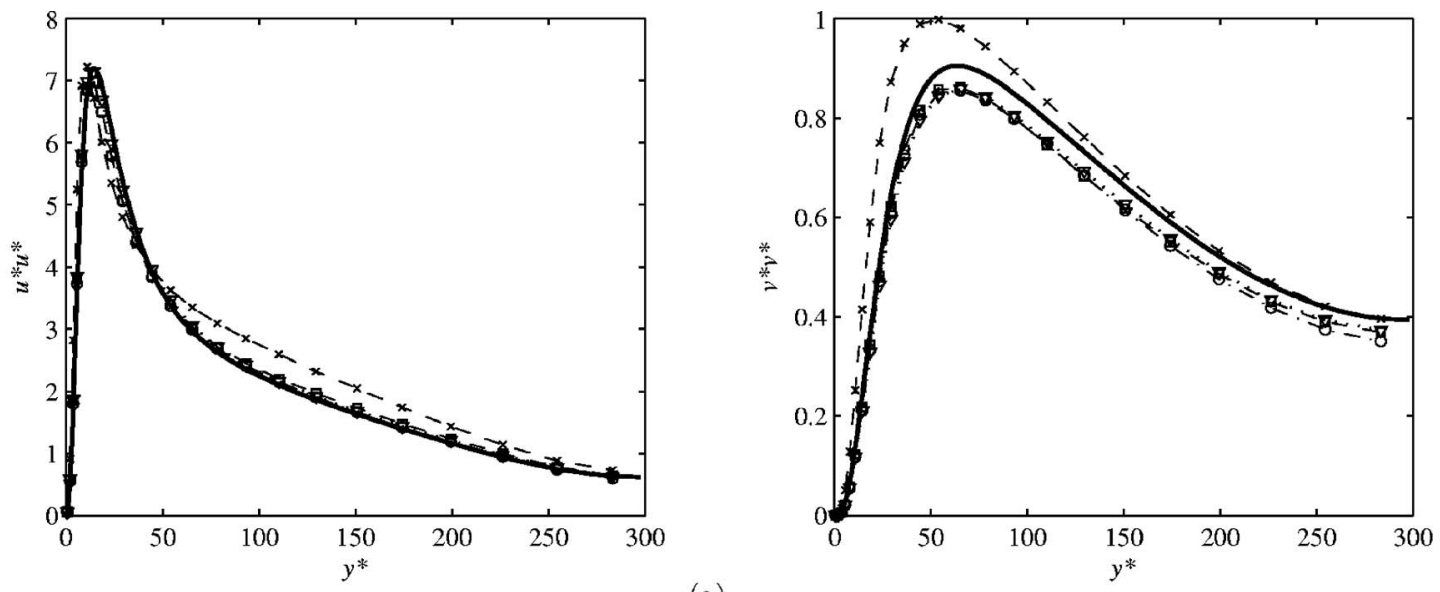

(a)
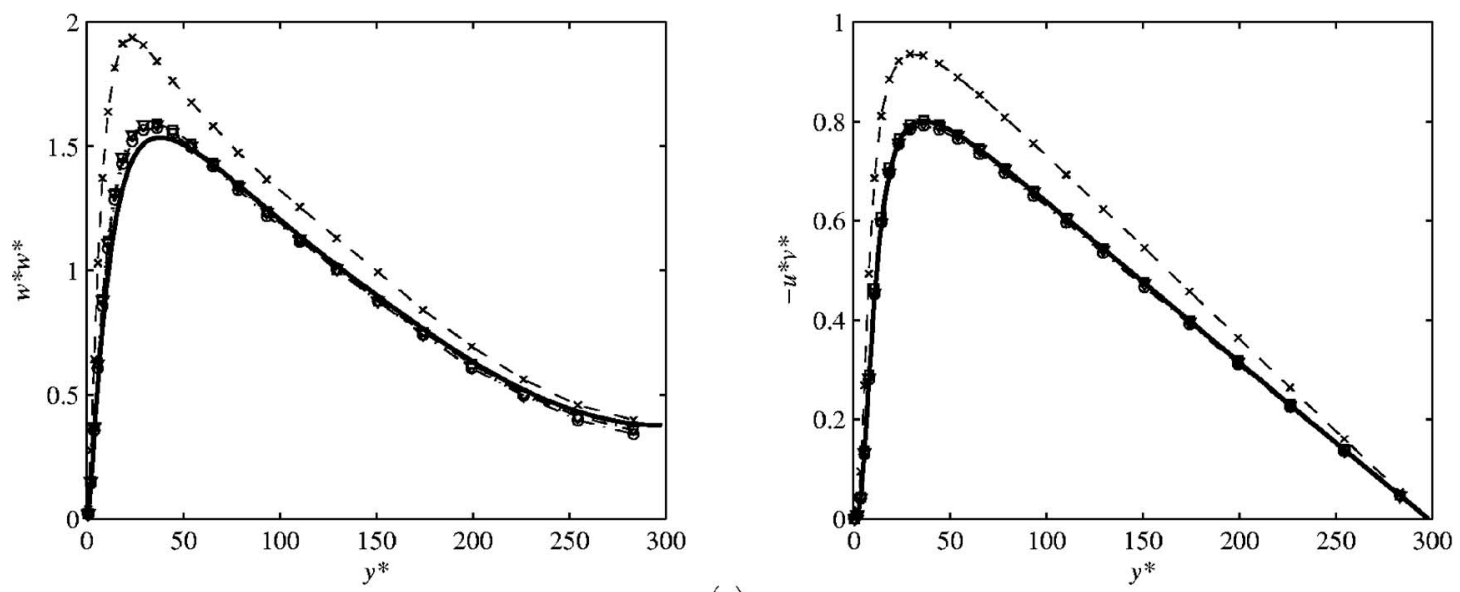

(c)

(d)

FIG. 7. Reynolds stresses $и и$ (a), $v v(\mathrm{~b}), w w$ (c), and $-u v$ (d), for the fitted models and the no-model simulation at $\mathrm{Re}_{\tau}=300$. ( $(-)$ : DNS reference; $(--\times)$ : no model; $(\cdots \nabla)$ : Model A; $(-. \circ)$ : Model B; and (-- $\square)$ : Model C.

trast, Model A, which has no filter-shape correction $\gamma_{\beta}$ in its formulation is very sensitive to such a change (variation of $6.4 \%)$.

In order to confirm these observations, and to exclude deceiving trends which may occur when only one flow prop-

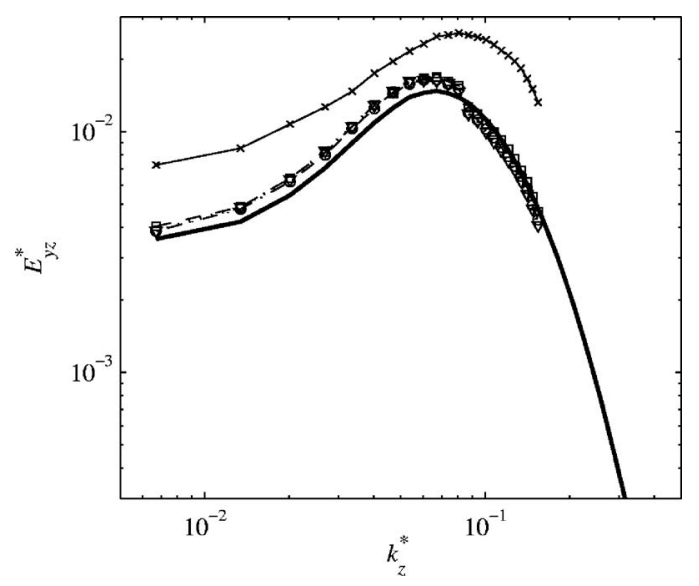

FIG. 8. Spanwise normal-velocity spectra $E_{y z}$ at $y^{*} \approx 15$, and $\operatorname{Re}_{\tau}=300$, for the fitted models and the no-model simulation. (-): DNS reference; $(--\times)$ : no model; $(\cdots \nabla)$ : Model A; $(-. \circ)$ : Model B; and $(--\square)$ : Model C. erty is considered in LES evaluations, ${ }^{16,18}$ we further evaluate the sensitivity of $u u, v v$, and $w w$ predictions. To this end, we look at the changes to the prediction of the respective maxima of the normal stresses. ${ }^{18}$ We define the sensitivity

$$
d_{u u}=\frac{\max \left(u u_{\text {sharp }}\right)-\max \left(u u_{\text {gauss }}\right)}{\max \left(u u_{\text {DNS }}\right)},
$$

with $u u_{\text {sharp }}$ and $u u_{\text {gauss }}$ the predictions obtained with $\mathcal{H}^{\prime}$, respectively, a sharp cutoff filter, and a Gauss filter. Similarly, $d_{v v}$, and $d_{w w}$ are defined. In Fig. 10, results are presented. Trends are clearly confirmed: Model A is much more sensitive to changes is the $\mathcal{H}^{\prime}$ filter shape than Models B and C. Model C is the least sensitive, with very stable predictions for $u u, v v$, and $w w$. In addition, for the sensitivity analysis in the remainder of this paper, we found no significant discrepancies between trends obtained from $\delta_{\tau}$ evaluations and $d_{u u}$, $d_{v v}$, and $d_{w w}$ trends, and results will be not further shown.

An evaluation of the models' sensitivity to changes in $\beta$ is presented in Fig. 11 for sharp cutoff high-pass filters and the three models A-C, and in Fig. 12 for Gaussian high-pass filters.

For sharp cutoff filters, Fig. 11 illustrates that all models are rather sensitive to changes in $\beta$. Including the $\beta=0$ case, the variation amounts to $19.4 \%$ for Model A, and $16.3 \%$ for 


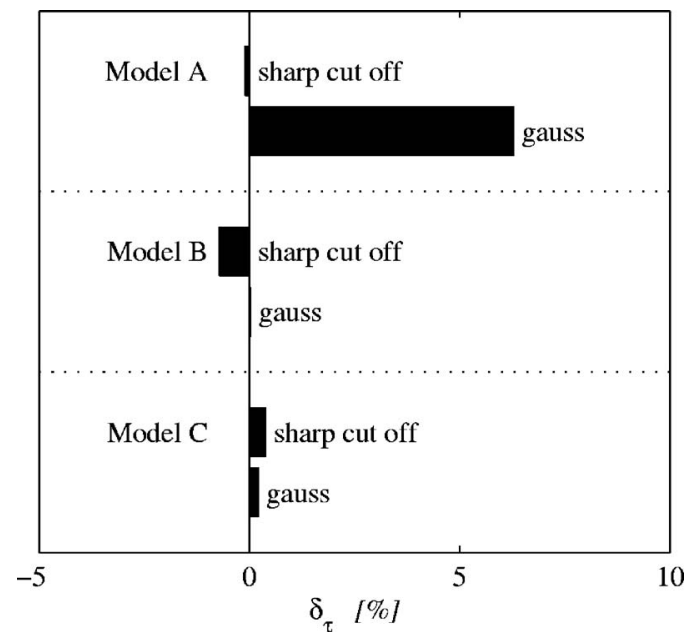

FIG. 9. Relative errors on the skin friction for different filters $\mathcal{H}^{\prime}, \operatorname{Re}_{\tau}$ $=300$ and $\beta=0.5$.

Models $\mathrm{B}$ and $\mathrm{C}$. The $\beta=0$ results correspond to normal Smagorinsky simulations cast into three variants A-C. However, in these Smagorinsky simulations, we excluded the mean-flow strain from the formulation of the model. Hence, these results represent the $\beta \rightarrow 0^{+}$limits of the respective small-small models. If we exclude the $\beta=0$ results, the variation observed for $0.25 \leqslant \beta \leqslant 0.75$ amounts to $14.3 \%$ for Model A, $13.9 \%$ for Model B, and $13.3 \%$ for Model C. These numbers can be compared with results on the sensitivity of the models using Gaussian high-pass filters in Fig. 12.

Compared to sharp cutoff results, the variations observed for Gaussian filters and $0.25 \leqslant \beta \leqslant 0.75$ (cf. Fig. 12) are significantly lower for Models B and C. They amount to $6.9 \%$ for Model B and 6.6\% for Model C. Results for Model A remain more or less the same, with a variation of $13.5 \%$. Hence, the use of Gaussian high-pass filters for small-small models improves their robustness when formulations $\mathrm{B}$ or $\mathrm{C}$ are used. This confirms similar observations which were obtained based on a theoretical analysis of these models. ${ }^{15}$

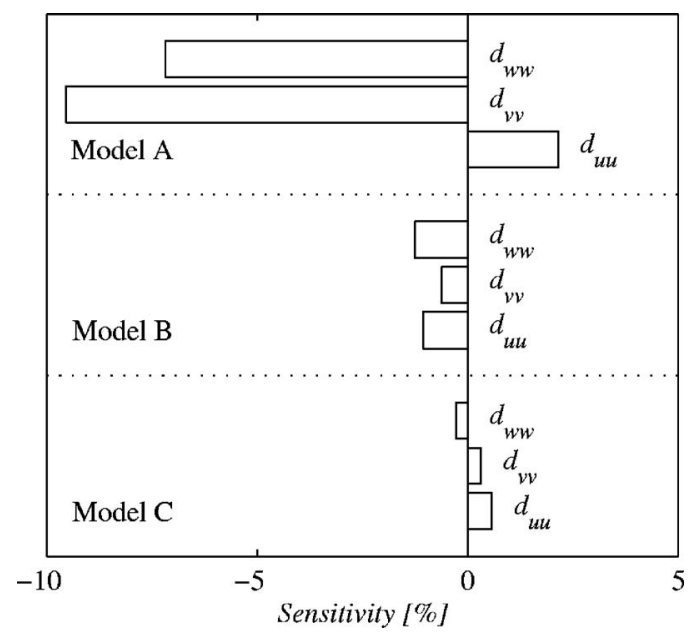

FIG. 10. Sensitivity of $\max (u u), \max (v v)$, and $\max (w w)$ to a change in the filter shape $\mathcal{H}^{\prime}$ from sharp cutoff to Gauss filter. $\operatorname{Re}_{\tau}=300$ and $\beta=0.5$.

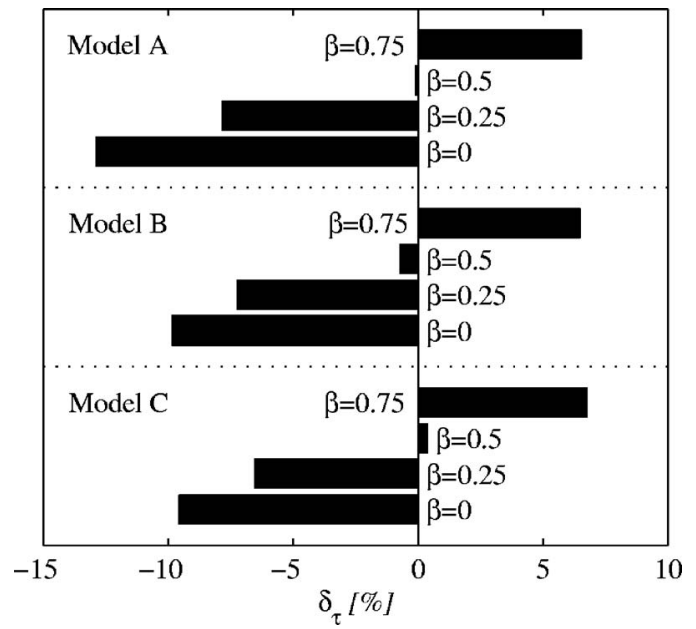

FIG. 11. Relative errors on the skin friction for different $\beta, \operatorname{Re}_{\tau}=300$, and $\mathcal{H}^{\prime}$ a sharp cutoff filter.

Next to higher robustness of simulations using Gaussian high-pass filters, we also observe that the jump in the spanwise normal-velocity spectra, as observed for sharp cutoff filters, disappears. This is shown in Fig. 13 for Model C, and the normal-velocity spanwise spectrum $E_{y z}$ at $y^{*} \approx 15$. In contrast to the sharp cutoff filter, the Gauss filter displays no discontinuity at $k_{c}^{\prime}$. Both filters yield LES results which correspond well with the DNS spectrum for $k_{z}^{*}>0.1$. The peak of the spectrum $\left(k_{z}^{*}<0.1\right)$ is overpredicted by both simulations.

\section{An evaluation of Reynolds-number effects}

We investigate how the three models react when $\mathrm{Re}_{\tau}$ varies. We recall, that it is our purpose to freeze possible effects related to the shape of $\mathcal{G}$ and to $R$, since we specifically aimed to exclude these uncertainties by calibrating the model coefficients. Therefore, the simulation grids for the different $\operatorname{Re}_{\tau}$ are selected such that the grid spacing, expressed in wall units, remains unchanged. In this way, varia-

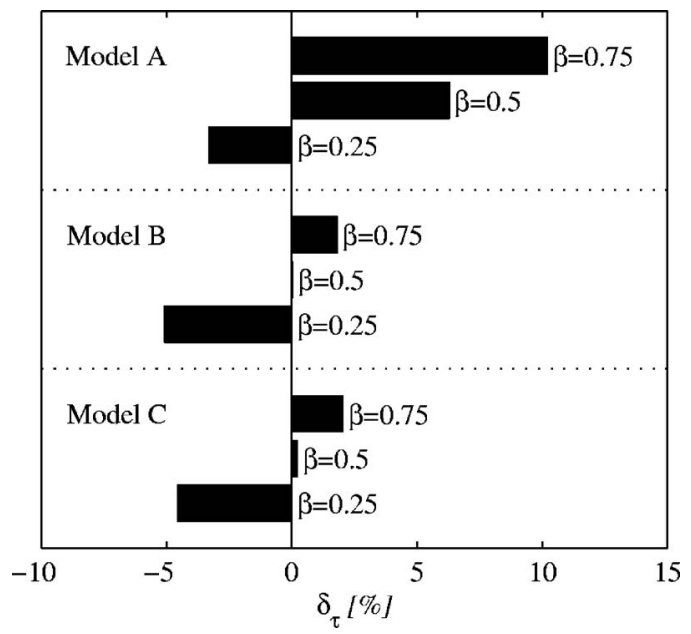

FIG. 12. Relative errors on the skin friction for different $\beta, \operatorname{Re}_{\tau}=300$, and $\mathcal{H}^{\prime}$ a Gauss filter. 


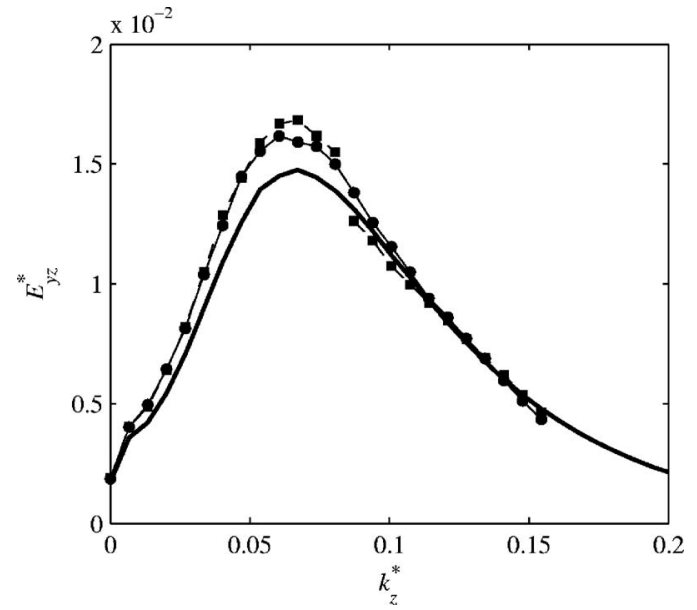

FIG. 13. Spanwise normal-velocity spectra $E_{y z}$ at $y^{*} \approx 15$, and $\operatorname{Re}_{\tau}=300$, for Model C. (-): DNS reference; $(--\mathbf{\square}): \mathcal{H}^{\prime}$ a sharp cutoff filter [Eq. (15)]; and (-••): $\mathcal{H}^{\prime}$ a Gaussian filter [Eq. (16)].

tions in $R$ and $\mathcal{G}$ close to the wall are small. An overview of the different simulation resolutions is presented in Table II.

In Fig. 14, we evaluate the variation on the skin-friction error $\delta_{\tau}$ for $\operatorname{Re}_{\tau}=110-650$. To better evaluate the variation around a common axis, the $\delta_{\tau}$ results of the three models are shifted with their averaged value over the different Reynolds numbers. For Models A, B, and C, these shifts in Fig. 14 correspond to $-0.19 \%,-0.18 \%$, and $-0.56 \%$. Results indicate that all three models are relatively insensitive to $\operatorname{Re}_{\tau}$ variations; i.e., all below $2 \%$. When the models are compared in more detail one can see that the variation on the skin-friction error is highest for Model A (1.9\%), followed by Model B (1.5\%), and lowest for Model C $(0.6 \%)$. Consequently, the inertial-range consistent formulation (Model C) seems best suited for an accurate fit over a wide range of Reynolds numbers; observed differences to the other two models are, however, not large enough to firmly establish this.

To further evaluate the quality of the simulation results, mean-velocity profiles $U(y)$ are presented in Fig. 15 for the $\mathrm{Re}_{\tau}=650$ case. As is appreciated, even at this elevated Rey-

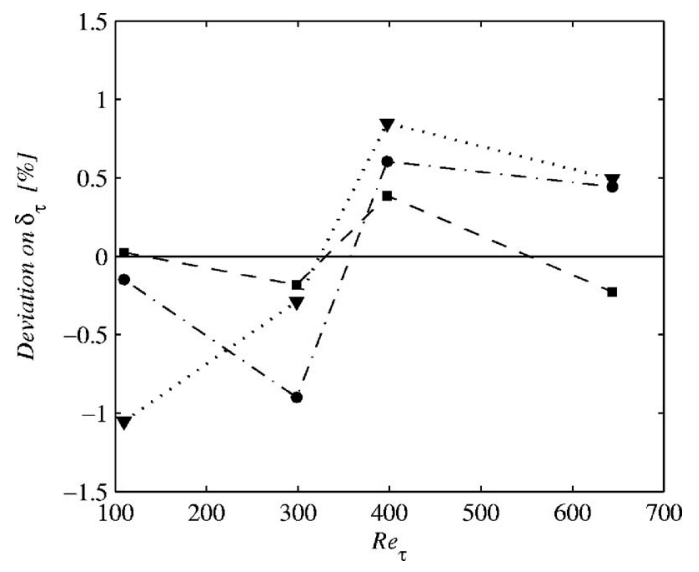

FIG. 14. Variation of $\delta_{\tau}$ as function of the Reynolds number for the $\operatorname{Re}_{\tau}$ $=300$-fitted models. $(\cdots \nabla)$ : Model A; $(-\cdot \bullet)$ : Model B; and $(--\mathbf{\square})$ : Model C.

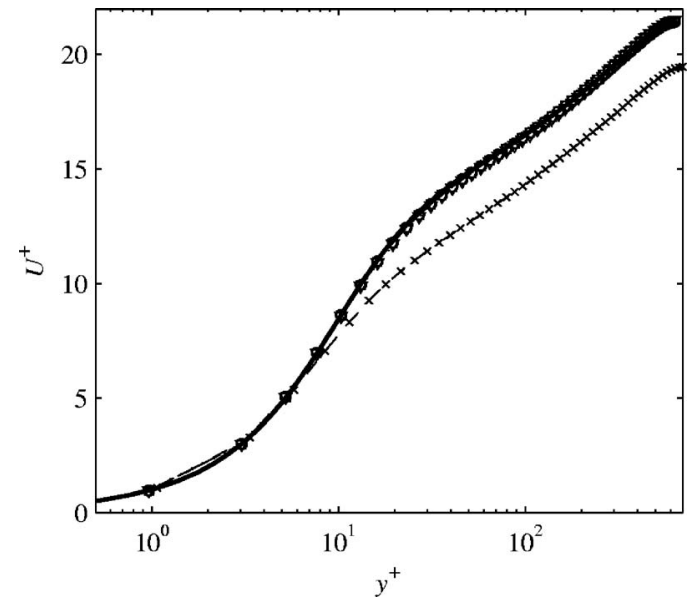

FIG. 15. Mean velocity profile for the different models and the no-model simulation at $\operatorname{Re}_{\tau}=650$. (-): DNS reference; $(--\times)$ : no model; $(\cdots \nabla)$ : Model A; (-.o): Model B; and (-- $\square)$ Model C.

nolds number, all three models predict $U(y)$ very well. In addition, the Reynolds stress predictions (not shown) are identical for the three models. Finally, the normal-velocity spanwise spectra are presented in Fig. 16 for $\operatorname{Re}_{\tau}=650$, and trends discussed in section IV B, e.g., the discontinuity at the $\mathcal{H}^{\prime}$ cutoff wavenumber, are again observed.

In studies of homogeneous isotropic turbulence, ${ }^{15-17}$ variations in the Re number typically lead to $\Delta / \eta$ changes. In these studies, it was shown that an inertial-rangeconsistent model formulation reduces simulation errors. Here, in plane channel flow simulations, Reynolds number variations do not lead to considerable differences between Model C and both other models. This is related to the gridselection in the current study. In order to keep $R$ and $\mathcal{G}$ effects small, the simulation grids close to the wall, expressed in wall units, were kept constant, leading to $\ell^{+} / \Delta^{+}$constant. However, as elaborated in Ref. 30, in the inner layer $\ell^{+} / \eta^{+}$ $\sim\left(y^{+}\right)^{3 / 4}$, and further independent of $\operatorname{Re}_{\tau}$. Hence, due to the current "wall-resolved" grid selections, a change in $\mathrm{Re}_{\tau}$ has no direct influence on the $\Delta / \eta$ ratio close to the wall.

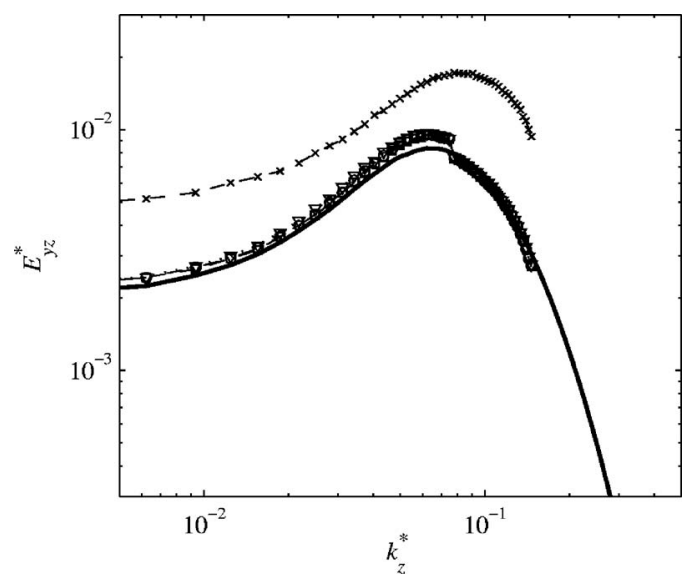

FIG. 16. Spanwise normal-velocity spectra $E_{y z}$ at $y^{*} \approx 15$, and $\operatorname{Re}_{\tau}=650$, for the different models and the no-model simulation. (-): DNS reference; $(--\times)$ : no model; $(\cdots \nabla)$ : Model A; $(-. \circ)$ : Model B; and $(--\square)$ : Model C. 
In the outer layer and the channel center $\Delta / \eta$ is not $\operatorname{Re}_{\tau}$ independent. However, also in the center of the channel, no significant differences appear in our simulations between the three models. This is related to the low local turbulent Reynolds number in the center of the channel. Based on the DNS data, ${ }^{19}$ we estimate that the Taylor-Reynolds number $\operatorname{Re}_{\lambda}$ in the channel center amounts to 14.6, 22.3, 25.6, and 32.7, for the respective $\operatorname{Re}_{\tau}=110,300$, 400, and 650 simulations. These numbers are very low: typical LES resolutions at these Reynolds numbers are very close to the minimum needed DNS resolution, such that the contribution of subgrid-scale models may be expected to be rather small. In addition, since we are using a structured grid, the $x$ and $z$ grid spacing close to the wall dictates a quite fine channel-center mesh. By evaluating the subgrid dissipation for the three models in our current simulations, we verified for all $\mathrm{Re}_{\tau}$ cases that the models' main contributions are situated in the near wall region up to the channel quarter-width $\delta / 2$.

\section{CONCLUSIONS}

We evaluated the performance of three variations of the small-small Smagorinsky model in the presence of walls. The classical small-small formulation ${ }^{9,11}$ (Model A) was augmented in two steps. The effects of the LES filter $\mathcal{G}$ and the high-pass filter $\mathcal{H}^{\prime}$ were explicitly incorporated in the formulation (Model B), and a fully inertial-range consistent formulation ${ }^{15}$ was considered (Model C).

We described four effects which may influence the performance of these models, and reflect on required levels of the model coefficients. These are (i) the influence of the LES filter shape $\mathcal{G}$, (ii) the influence of the high-pass filter $\mathcal{H}^{\prime}$, (iii) finite-Reynolds-number effects expressed in finite $\Delta / \eta$ ratios, and (iv) a low resolution $R$ of the dominant turbulent scales. Models B and C were designed to compensate filtershape effects and finite-Reynolds-number effects. Therefore, the effect of changes related to items (ii) and (iii) have been investigated during this study, quantifying the improvements of Models B and C compared to Model A in turbulent channel flows. Uncertainties related to (i) and (iv) were limited by first performing a set of calibration runs at $\operatorname{Re}_{\tau}=300$, and then keeping effects related to $\mathcal{G}$ and $R$ constant during the sensitivity analysis of other parameters.

Changes to the high-pass filter shape $\mathcal{H}^{\prime}$ have a profound effect. Sharp cutoff filters and Gaussian filters were investigated. Keeping $\beta=\Delta / \Delta^{\prime}$ constant, Models B and C were rather insensitive to changes from a sharp cutoff filter to a Gaussian filter, with a variation on skin-friction predictions of $0.16 \%$ (Model C) and $0.74 \%$ (Model B). Model A, which lacks the explicit inclusion of filter effects in its formulation, displayed a much larger variation of $6.4 \%$.

When $\beta=\Delta / \Delta^{\prime}$ is varied, conclusions are different for the two filter types which were considered. For sharp cutoff high-pass filters, all models show a large dependence on $\beta$. When Gaussian filters are employed, Models B and C were shown to be rather insensitive to variations in $\beta$. Model A again displayed large changes in simulation quality.

Finally, all simulations with accurate skin-friction predictions provided, independently of the model formulation, very good results for the mean-velocity profiles and the Reynolds stresses. The spanwise normal-velocity spectra were also well predicted, but here some differences in results were observed between simulations using sharp cutoff and Gaussian high-pass filters. The use of a sharp cutoff filters is visible in the spectrum as a discontinuity at the high-pass cutoff wavenumber. For Gauss filters, such a discontinuity does not appear.

By varying the Reynolds number, i.e., $\operatorname{Re}_{\tau}=110,300$, 400 , and 650 were considered, we observed that all three models are relatively insensitive to changes in the Reynolds number. Errors on the skin-friction prediction remain within $2 \%$. Of the three models, Model C shows the smallest sensitivity, with a variation on the prediction of the skin-friction of about $0.6 \%$. The relative insensitivity of all three the models to $\operatorname{Re}_{\tau}$ variations was hypothesized to be connected to our structured-grid discretization, and the low turbulent Reynolds numbers in the channel center. Therefore, channel-flow simulations at higher Reynolds numbers using an unstructured discretization may be interesting for future research.

In the present work it was shown that the theoretical improvements of small-small Smagorinsky models obtained for locally isotropic turbulence, ${ }^{15}$ also reflect on channelflow simulation results. By incorporating filter-shape, and finite-Reynolds-number effects explicitly in the formulation of the models, the level of empiricism typically encountered when model coefficients are selected, is reduced.

An essential part of the evaluations of the three model formulations $(\mathrm{A}-\mathrm{C})$ has been their initial calibration. In practice, validation or calibration is required for any subgridscale closure and discretization in LES. In this sense, the models in the current paper are no exception. However, we exploited this calibration to exclude uncertain effects such as the filter shape $\mathcal{G}$ and the ratio $R=\ell / \Delta$, and to define a consistent platform to compare high-pass-filter and $\operatorname{Re}_{\tau}$ effects. This allowed a precise evaluation of the models' portability, e.g., subject to high-pass filter changes, and illustrated the potential of Models B and C, compared to Model A. A logical next step concentrates on means to incorporate the remaining $\mathcal{G}$ and $R$ effects in the VMS formulations, or to identify working conditions for which they can be disregarded. This is currently a topic of ongoing research.

${ }^{1}$ P. Sagaut, Large Eddy Simulations for Incompressible Flows, 3rd ed. (Springer, Berlin, 2006).

${ }^{2}$ R. S. Rogallo and P. Moin, "Numerical simulations of turbulent flows," Annu. Rev. Fluid Mech. 16, 99 (1984).

${ }^{3}$ M. Lesieur and O. Métais, "New trends in large-eddy simulations of turbulence," Annu. Rev. Fluid Mech. 28, 45 (1996).

${ }^{4}$ B. J. Geurts, Elements of Direct and Large-Edddy Simulation (Edwards, Flourtown, 2003).

${ }^{5}$ C. Meneveau, "Statistics of turbulence subgrid-scale stresses: Necessary conditions and experimental tests," Phys. Fluids 6, 815 (1994).

${ }^{6} \mathrm{H}$. Suk Kang, S. Chester, and C. Meneveau, "Decaying turbulence in an active-grid-generated flow and comparisons with large-eddy simulation," J. Fluid Mech. 480, 129 (2003).

${ }^{7}$ S. B. Pope, Turbulent Flows (Cambridge University Press, Cambridge, UK, 2000).

${ }^{8}$ T. J. R. Hughes, A. A. Oberai, and L. Mazzei, "Large eddy simulation of turbulent channel flows by the variational multiscale method," Phys. Fluids 13, 1784 (2001). 
${ }^{9}$ T. J. R. Hughes, L. Mazzei, and K. E. Jansen, "Large eddy simulation and the variational multiscale method," Comput. Visualization Sci. 3, 47 (2000).

${ }^{10}$ J. Smagorinsky, "General circulation experiments with the primitive equations: I. The basic experiment," Mon. Weather Rev. 91, 99 (1963).

${ }^{11}$ T. J. R. Hughes, L. Mazzei, and A. A. Oberai, "The multiscale formulation of large eddy simulation: Decay of homogeneous isotropic turbulence," Phys. Fluids 13, 505 (2001).

${ }^{12}$ H. Jeanmeart and G. S. Winckelmans, "Comparison of recent dynamic subgrid-scale models in turbulent channel flow," in Studying Turbulence Using Numerical Simulation Databases IX (CTR, Stanford, 2002), pp. $105-116$.

${ }^{13}$ J. Holmen, T. J. R. Hughes, A. A. Oberai, and G. N. Wells, "Sensitivity of the scale partition for variational multiscale large-eddy simulation of channel flow," Phys. Fluids 16, 824 (2004).

${ }^{14}$ S. Stolz, P. Schlatter, and L. Kleiser, "High-pass filtered eddy-viscosity models for large-eddy simulations of transitional and turbulent flow," Phys. Fluids 17, 065103 (2005).

${ }^{15}$ J. Meyers and P. Sagaut, "On the model coefficients for the standard and the variational multi-scale Smagorinsky model,” J. Fluid Mech. 569, 287 (2006).

${ }^{16}$ J. Meyers, P. Sagaut, and B. J. Geurts, "Optimal model parameters for multi-objective large-eddy simulations," Phys. Fluids 18, 095103 (2006).

${ }^{17}$ J. Meyers and P. Sagaut, "On the required Reynolds-number dependence of variational multi-scale Smagorinsky models," in Proceedings of ECCOMAS CFD 2006, edited by P. Wesseling, E. Oñate, and J. Périaux, September 5-8, Egmond aan Zee, The Netherlands (TU Delft, 2006), ISBN 90-9020970-0, Art. No. 439.

${ }^{18}$ J. Meyers and P. Sagaut, "Is plane-channel flow a friendly case for the testing of large-eddy simulation subgrid-scale models?" Phys. Fluids 19, 048105 (2007).

${ }^{19}$ K. Iwamoto, Y. Suzuki, and N. Kasagi, "Reynolds number effect on wall turbulence: toward effective feedback control," Int. J. Heat Fluid Flow 23, 678 (2002); see also http://www.thtlab.t.u-tokyo.ac.jp/DNS/ dns_database.html

${ }^{20}$ M. Frigo and S. G. Johnson, "The design and implementation of FFTW3,"
Proc. IEEE 93, 216 (2005); see also "Fastest Fourier Transform in the West," http://www.fftw.org/

${ }^{21}$ R. W. C. P. Verstappen and A. E. P. Veldman, "Symmetry-preserving discretization of turbulent flow," J. Comput. Phys. 187, 343 (2003).

${ }^{22}$ C. Hirsch, Fundamentals of Numerical Discretization, Numerical Computation of Internal and External Flows, Vol. 1 (John Wiley \& Sons, New York, 1988).

${ }^{23}$ G. S. Winckelmans and H. Jeanmeart, "Assessment of some models for LES without/with explicit filtering," in Direct and Large-Eddy Simulation IV (Kluwer, Dordrecht, 2001), p. 55.

${ }^{24}$ A. W. Vreman, "The filtering analog of the variational multiscale method in large-eddy simulation," Phys. Fluids 15, L61 (2003).

${ }^{25}$ A. W. Vreman, "The adjoint filter operator in large-eddy simulation of turbulent flow," Phys. Fluids 16, 2012 (2004).

${ }^{26}$ D. K. Lilly, "The representation of small-scale turbulence in numerical simulation experiments," in Proceedings of the IBM Scientific Computing Symposium on Environmental Sciences (IBM Data Processing Division, White Plains, 1967).

${ }^{27}$ J. W. Deardoff, "A numerical study of three-dimensional turbulent channel flow at large Reynolds numbers," J. Fluid Mech. 41, 453 (1970).

${ }^{28}$ A. Muschinski, "A similarity theory of locally homogeneous and isotropic turbulence generated by a Smagorinsky-type LES," J. Fluid Mech. 325, 239 (1996).

${ }^{29}$ J.-C. Magnient, P. Sagaut, and M. Deville, "A study of built-in filter for some eddy viscosity models in large-eddy simulation," Phys. Fluids 13, 1440 (2001).

${ }^{30}$ H. Tennekes and J. L. Lumley, A First Course in Turbulence, 5 th printing (MIT, Cambridge, 1978), p. 159.

${ }^{31}$ A. Scotti, C. Meneveau, and D. K. Lilly, "Generalized Smagorinsky model for anisotropic grids," Phys. Fluids A 5, 2306 (1993).

${ }^{32}$ G. S. Winckelmans, H. Jeanmart, and D. Carati, "On the comparison of turbulence intensities from large-eddy simulation with those from experiment or direct numerical simulation," Phys. Fluids 14, 1809 (2002).

${ }^{33}$ P. Sagaut and V. Levasseur, "Sensitivity of spectral variational multiscale methods for large-eddy simulation of isotropic turbulence," Phys. Fluids 17, 035113 (2005). 